\title{
La flore exotique potentiellement envahissante d'Algérie : première description des espèces cultivées en pépinières et dans les jardins
}

\author{
Nora Sakhraoui ${ }^{(1)}$, Sofia Metallaoui ${ }^{(1)}$, Azzedine Chefrour ${ }^{(2)}$, Azzedine Hadef ${ }^{(1)}$ \\ (1) Université 20 Aout 1955. Faculté des Sciences. Département des Sciences de la Nature et de la Vie (SNV). BP 26. \\ Route d'El Hadaiek. 21000 Skikda (Algérie).E-mail : sakhraouinora05@gmail.com \\ (2) Université Mohamed Cherif Messaadia. Faculté des Sciences de la Nature et de la Vie. Département de Biologie. \\ 41000 Souk Ahras (Algérie).
}

Reçu le 28 mai 2018, accepté le 4 février 2019, mis en ligne le 5 avril 2019.

Cet article est distribué suivant les termes et les conditions de la licence CC-BY (http://creativecommons.org/licenses/by/4.0/ deed.fr)

Description du sujet. La flore exotique potentiellement envahissante cultivée dans la wilaya de Skikda (nord-est algérien) n'a jamais fait l'objet de recherche. Vu les effets néfastes potentiels qu'elle exercerait sur les écosystèmes si elle est avérée envahissante, une étude a été réalisée dans 60 localités horticoles réparties sur 4 pépinières, 54 jardins privés et 2 jardins de l'Université de Skikda.

Objectifs. Cette étude vise à déterminer les espèces potentiellement envahissantes cultivées dans ces localités, étudier leurs caractéristiques et estimer leur degré de naturalisation.

Méthodes. La liste des espèces potentiellement envahissantes a été élaborée sur la base des listes internationales figurant dans différents travaux. Les relevés floristiques effectués mentionnent le type biologique et les éventuelles fructification et régénération des plantules suivis in situ. Le degré de naturalisation a été déterminé grâce à la réalisation de prospections au niveau de trois communes.

Résultats. Cinquante espèces et 43 genres appartenant à 29 familles botaniques ont été identifiés. Les familles les mieux représentées sont les Fabaceae, les Cactaceae, les Myrtaceae et les Poaceae. Les espèces d'origine américaine sont largement dominantes (46\%), suivies des espèces asiatiques (26\%), australiennes (20\%) et enfin celles de l'Afrique australe (8\%). Tous les types biologiques ont été enregistrés avec une dominance des espèces ligneuses (66\%). La fructification a été observée chez 40 espèces dont 13 seulement parviennent à produire des plantules dans le voisinage des pieds mères. Onze espèces sont naturalisées dans les sites prospectés, 2 en cours de naturalisation et 37 non naturalisées.

Conclusions. Pour la première fois dans la région, la liste préliminaire des espèces potentiellement envahissantes cultivées est établie, ce qui devrait permettre la mise en place d'un plan d'action visant à surveiller leur culture et leur commercialisation. Mots-clés. Espèces introduites, espèces envahissantes, enquête, Algérie.

The potentially invasive alien flora of Algeria: first description of potentially invasive species cultivated in nurseries and gardens

Description of the subject. The potentially invasive alien flora cultivated in the wilaya of Skikda has never previously been the subject of research. In view of the adverse effects this flora has on ecosystems if it becomes invasive, a study was carried out in 60 horticultural localities, spread over 4 nurseries, 54 private gardens and 2 gardens of Skikda University.

Objectives. This study aimed to determine the potentially invasive species cultivated in these localities, to study their characteristics, and to estimate their degree of naturalization.

Method. A list of potentially invasive species was elaborated on the basis of the international lists appearing in various existing studies. The floristic records specify the biological type and the possible fruiting and regeneration of the seedlings followed in situ. The degree of naturalization of the species was determined by conducting surveys in three municipalities.

Results. Fifty species and 43 genera, belonging to 29 botanical families, were identified. The best represented families were Fabaceae, Cactaceae, Myrtaceae and Poaceae. Species of American origin were largely dominant (46\%), followed by Asian 
(26\%) and Australian (20\%) species, and finally, by those of southern African (8\%) origin. All biological types were recorded, with a dominance shown by woody species (66\%). Fruiting was observed in 40 species, only 13 of which managed to produce seedlings in the vicinity of the mother plants. Eleven species were naturalized in the sites prospected, 2 were in the process of naturalization, and 37 were non-naturalized species.

Conclusions. For the first time in the region, a preliminary list of potentially invasive species cultivated has been established. This should allow the implementation of an action plan to monitor the cultivation and commercialization of these species.

Keywords. Introduced species, invasive species, surveys, Algeria.

\section{INTRODUCTION}

L'invasion des espèces végétales est une préoccupation mondiale. Aucun pays n'est réellement à l'abri de ce problème à cause de la multiplication des échanges commerciaux et des difficultés rencontrées pour la mise en place de systèmes de contrôle efficaces du matériel végétal transitant via les multiples moyens de transport. La filière horticole est une voie majeure d'introduction d'espèces exotiques et invasives dans le monde (Reichard \& White, 2001 ; Dehnen-Schmutz et al., 2007).

En effet, certaines espèces exotiques parviennent à se naturaliser dans la zone d'introduction et peuvent devenir envahissantes. L'Union Internationale pour la Conservation de la Nature (UICN) définit une espèce envahissante comme une espèce exotique qui s'implante dans un habitat naturel ou semi-naturel, induit des changements et menace la diversité biologique indigène (Shine et al., 2000). Ces espèces s'installent le plus souvent dans les milieux perturbés où elles peuvent être indicatrices de cette perturbation.

Les espèces invasives sont un véritable danger pour la biodiversité via la réduction de la richesse spécifique (Vilà et al., 2015), la pollution génétique et l'érosion génétique. Elles sont d'ailleurs considérées comme la deuxième cause d'érosion de la biodiversité après la destruction et la fragmentation des habitats (OTA, 1993).

En Algérie, les invasions végétales ont été peu étudiées, parmi les travaux réalisés il convient de citer ceux de Le Floc'h et al. (1990), Véla et al. (2013) et Meddour \& El Mokni (2016). La flore exotique à potentiel invasif n'est pas identifiée et les listes d'espèces envahissantes ou à surveiller, comme celles établies dans certains pays méditerranéens tels que la France (Brunel \& Tison, 2005), l'Espagne (Capdevilla Argüelles et al., 2006) et l'Italie (Celesti-Grapow et al., 2009a), sont inexistantes en Algérie. C'est dans ce contexte que nous avons mené cette étude dans la wilaya de Skikda située au nord-est de l'Algérie, qui connait depuis quelques années un développement notable dans le secteur de l'horticulture. Des relevés floristiques ont été réalisés dans des pépinières et des jardins afin d'identifier la flore exotique à potentiel invasif cultivée et commercialisée sur le territoire de la wilaya. Nous rapportons la richesse spécifique, l'origine des espèces recensées ainsi que quelques caractéristiques observées concernant essentiellement les aptitudes à la fructification et à la régénération de plantules. Ce travail, bien qu'il ne soit pas exhaustif, vise à actualiser les données relatives aux plantes exotiques cultivées en Algérie. Il peut constituer une base pour la mise en place d'un système de surveillance visant à contrôler la commercialisation et la propagation des espèces exotiques envahissantes ou à potentiel invasif.

\section{MATÉRIEL ET MÉTHODES}

\subsection{Description du site d'étude}

La wilaya de Skikda est située au nord-est de l'Algérie. Elle est limitée au nord par la mer Méditerranée, au sud-est par la wilaya de Guelma, au sud-ouest par les wilayas de Constantine et Mila, à l'est par la wilaya d'Annaba et à l'ouest par la wilaya de Jijel. Elle s'étend sur une superficie de $4140 \mathrm{~km}^{2}$, découpée administrativement en 13 daïras et 38 communes (Meghzili, 2015) (Figure 1). En appliquant la méthode d'Emberger (1955), qui permet de caractériser le type de climat en Méditerranée, la région de Skikda peut être classée dans l'étage bioclimatique humide à subhumide. L'étage humide couvre la zone occidentale montagneuse représentée par le massif de Collo (De Belair \& Sameraoui, 2000), l'étage subhumide couvre le reste de la wilaya (Farah, 2014).

Les jardins privés ont été sélectionnés de façon aléatoire à partir d'une liste de 110 jardins distribués sur la zone d'étude, auxquels nous pouvions accéder. Situés majoritairement dans des zones périurbaines, ils se répartissent dans quatre communes : 37 jardins sont localisés dans la Cité Larbi Ben M'Hidi (commune de Skikda), 5 jardins dans la commune de Filfilla, 6 jardins dans la commune d'El Hadaiek et 6 jardins dans la commune de Hamadi Krouma. Ces jardins sont généralement exploités pour les cultures saisonnières, notamment maraichères ou en arboriculture fruitière ; les plantes ornementales sont occasionnellement cultivées en guise de haies, brise-vent ou d'ornement.

Les jardins de l'Université 20 Aout 1955 de Skikda (jardin botanique et jardin mexicain) sont très anciens. Ils comptent parmi les vestiges de l'ancienne école d'agriculture de Philippeville créée en 1900 et font 


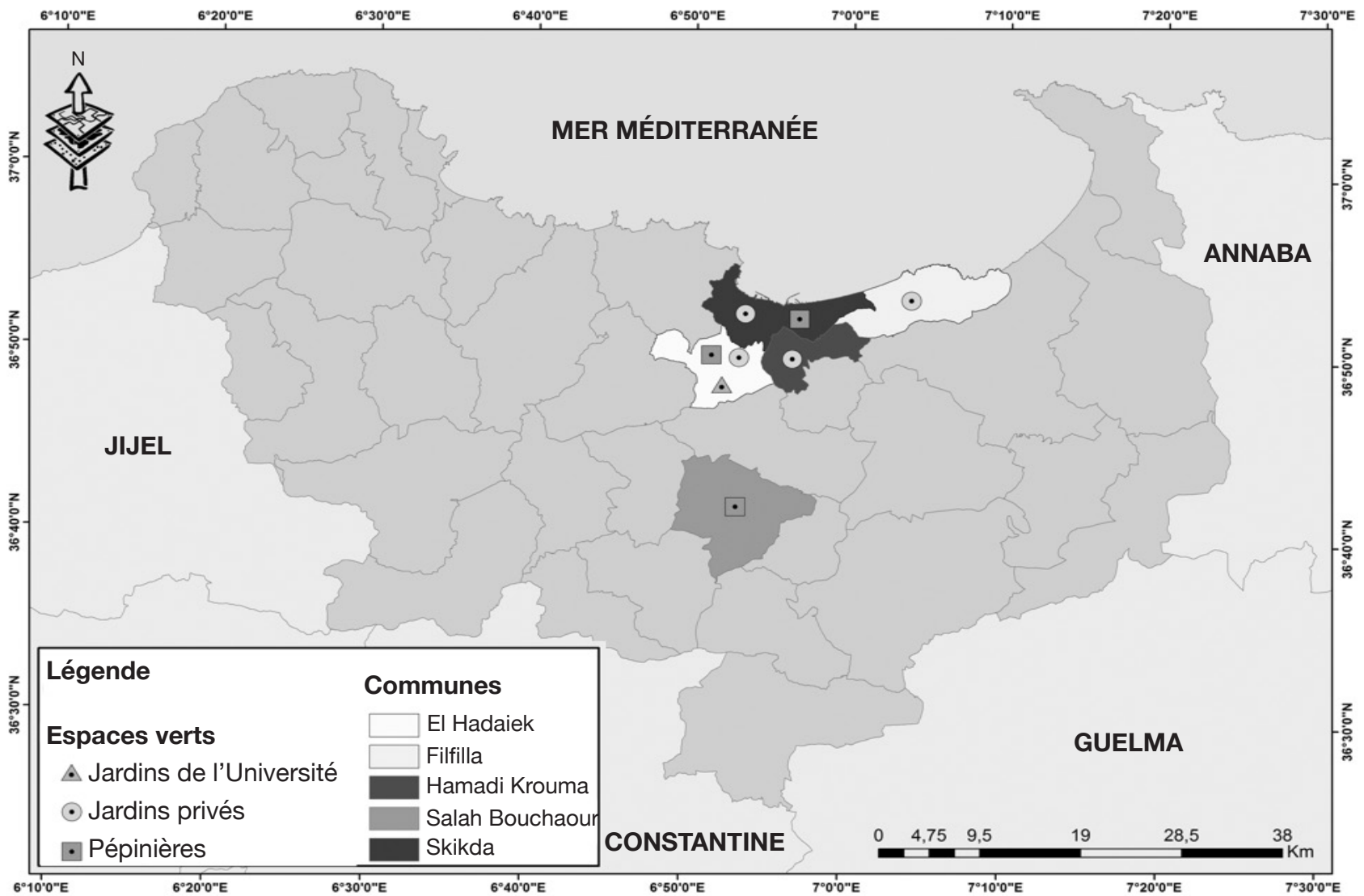

Figure 1. Localisation des sites prospectés - Localisation of sites prospected.

partie d'un ensemble d'espaces verts, situé dans la partie ouest du campus universitaire et dont la superficie totale avoisine 1,5 ha. Le campus universitaire se trouve à $4 \mathrm{~km}$ au sud-ouest de la ville de Skikda, sur la route d'El Hadaiek.

Les pépinières sont toutes installées en bordure des routes principales dans des zones périurbaines. Elles se répartissent dans trois communes : vergers d'Algérie pour l'aménagement des espaces verts (commune de Skikda), pépinière El Hadaiek 1, pépinière El Hadaiek 2 (commune d'El Hadaiek) et la pépinière Mokhbi (commune de Salah Bouchaour).

\subsection{Méthodologie de travail}

Entre 2014 et 2018, des dizaines de visites ont été réalisées au niveau des 4 pépinières, des 54 jardins privés et des jardins de l'Université de Skikda (jardin botanique et jardin mexicain). Au total, 60 localités horticoles ont été prospectées.

À partir de l'inventaire des espèces cultivées, la liste des espèces potentiellement envahissantes dans notre région a été élaborée sur la base des listes étrangères des espèces considérées comme envahissantes dans les différentes parties du monde. Les listes consultées sont celles parues dans les travaux de Muller (2000), Brunel
\& Tison (2005), Capdevilla Argüelles et al. (2006), Fourdrigniez \& Meyer (2008), Celesti-Grapow et al. (2009a ; 2009b), Hequet et al. (2009), Arianoutsou et al. (2010), Moran et al. (2013), Terrin et al. (2014) et Weber (2017).

La nomenclature adoptée pour les Cactaceae est celle proposée par Véla (2013) ; pour les autres taxons, comme il s'agit d'espèces introduites généralement absentes des flores traitant la végétation autochtone, la nomenclature adoptée est celle parue dans des ouvrages spécialisés en espèces horticoles comme Bruni et al. (2006), Odenwald \& Turner (2006) et Cullen et al. (2011). La présentation des familles végétales d'Angiospermes est réalisée selon l'Angiosperme Phylogeny Group (APG III, 2009). Des relevés floristiques concernant le type biologique déterminé selon Raunkiaer (1934), le mode de reproduction, la fructification et l'établissement de nouvelles plantules issues de la reproduction sexuée ont été effectués. Ces deux caractères sont suivis in situ, ils marquent la capacité des espèces à se régénérer sexuellement sans l'intervention de l'homme. La richesse spécifique et la richesse générique sont exprimées par le nombre total d'espèces et des genres recensés.

Enfin, nous avons réalisé une enquête au niveau des communes de Skikda, Filfilla et El Hadaiek pour 
déterminer le degré de naturalisation des espèces figurant dans la liste établie. L'accessibilité des sites par voie routière était un critère majeur dans la sélection des zones prospectées. Le degré de naturalisation a été estimé selon Magnanon et al. (2008). Les statuts retenus sont :

- espèce naturalisée : espèce non indigène, mêlée à la flore indigène et se maintenant dans les stations d'observation depuis plus de quinze années consécutives ;

- espèce en voie de naturalisation : espèce non indigène apparue en milieu naturel depuis environ cinq ans et formant des populations stabilisées ;

- espèce non naturalisée : espèce non indigène cultivée n'ayant pas échappé aux cultures.

\section{RÉSULTATS}

\subsection{Aspect systématique}

Au sein de la flore exotique cultivée dans les différents sites sélectionnés, 29 familles regroupant des espèces potentiellement envahissantes dans notre territoire appartenant toutes aux Angiospermes ont été recensées (Tableau 1). Les familles les mieux représentées sont les Fabaceae (sept espèces), les Cactaceae (cinq espèces), les Myrtaceae (quatre espèces), les Poaceae (quatre espèces), les Solanaceae (trois espèces), les Verbenaceae, les Bignoniaceae et les Commelinaceae, chacune représentée par deux espèces. Les autres familles sont représentées par une seule espèce.

La flore introduite recensée comprend 43 genres et 50 espèces. Les genres les mieux représentés sont Acacia (quatre espèces), Opuntia (trois espèces) et Psidium (deux espèces). Les Eudicotylédones sont les plus abondantes (40 espèces), représentées par 27 espèces dans les jardins de l'Université, 19 espèces dans les pépinières et 21 espèces dans les jardins privés. Les Monocotylédones sont moins nombreuses (10 espèces seulement), 10 espèces ont été recensées dans les jardins de l'Université et 7 espèces dans les pépinières et les jardins privés.

Trente-sept espèces ont été répertoriées dans les jardins de l'Université de Skikda, 28 espèces dans les différents jardins privés et enfin 26 espèces ont été répertoriées dans les pépinières (Figure 2).

Parmi les espèces végétales recensées, signalées envahissantes dans les différentes régions du monde, 15 espèces $(30 \%)$ n'ont été trouvées que dans les jardins de l'Université, quatre espèces $(8 \%)$ n'ont été observées que dans les pépinières: Brugmansia suaveolens, Leucaena leucocephala, Macfadyena unguis-cati et Salix babylonica et six espèces (12\%) ne sont présentes que dans les jardins privés: Acacia horrida, Ailanthus altissima, Carpobrotus edulis,
Nicotiana glauca, Opuntia stricta et Phytolacca americana. Enfin, 15 espèces (30\%) sont retrouvées dans les trois types de localités concernées par cette étude.

\subsection{Aspect biologique}

Les espèces dénombrées appartiennent à des types biologiques différents (Figure 3). La dominance des espèces ligneuses est nettement marquée par un pourcentage de $66 \%$.

En ce qui concerne la reproduction, les espèces peuvent être classées selon trois modes : 23 espèces se reproduisent à la fois par le mode végétatif et sexué, 10 espèces se multiplient uniquement de manière végétative et 17 espèces se reproduisent exclusivement de façon sexuée.

\subsection{Aspect physiologique}

Le suivi in situ des aptitudes à la fructification et à la régénération des plantules issues de graines, proliférant dans le voisinage des plantes mères (Tableau 2), a montré que 40 espèces $(82 \%)$ parviennent à produire des fruits dans les différents sites d'étude.

Parmi ces 40 espèces, seulement 13 espèces $(31,70 \%)$ présentent des plantules dans le voisinage des pieds mères.

\subsection{Origine géographique}

L'étude de l'origine des espèces recensées révèle que les espèces d'origine américaine sont les plus abondantes (46\%), suivies des espèces asiatiques $(26 \%)$, des espèces australiennes $(20 \%)$ et enfin des espèces de l'Afrique australe $(8 \%)$.

\subsection{Degré de naturalisation}

La prospection dans les trois communes sélectionnées a permis de déterminer trois degrés de naturalisation : 11 espèces sont naturalisées, deux espèces sont en cours de naturalisation et 37 espèces sont non naturalisées (Tableau 2).

\section{DISCUSSION}

Le jardin botanique et le jardin mexicain de l'Université de Skikda abritent une collection végétale au sein de laquelle plusieurs espèces potentiellement envahissantes ont été recensées, ils se distinguent des autres sites par le nombre important d'espèces potentiellement envahissantes enregistrées ainsi que par le taux d'espèces proliférant exclusivement à leur niveau. Les jardins botaniques jouent un rôle important 


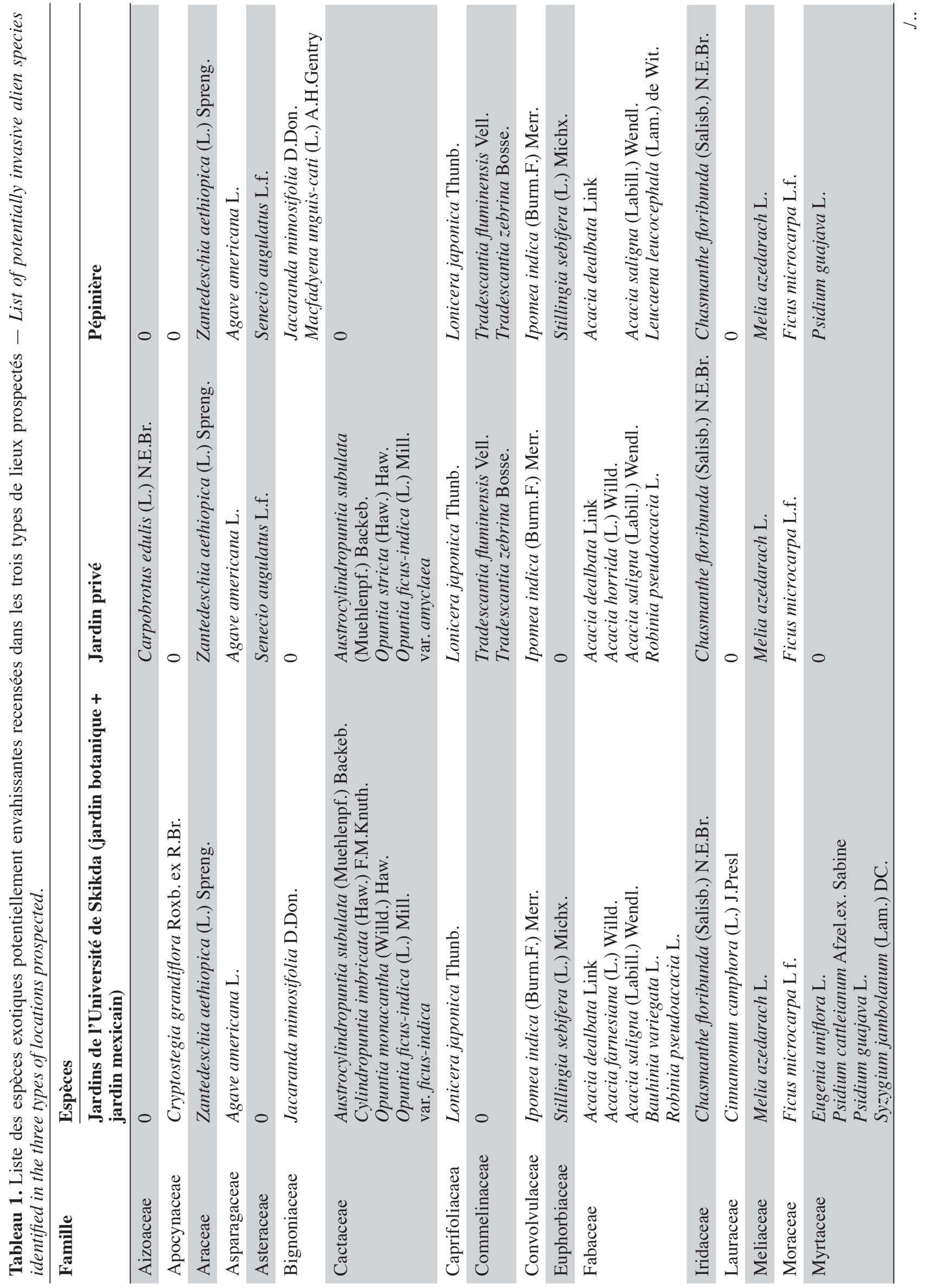



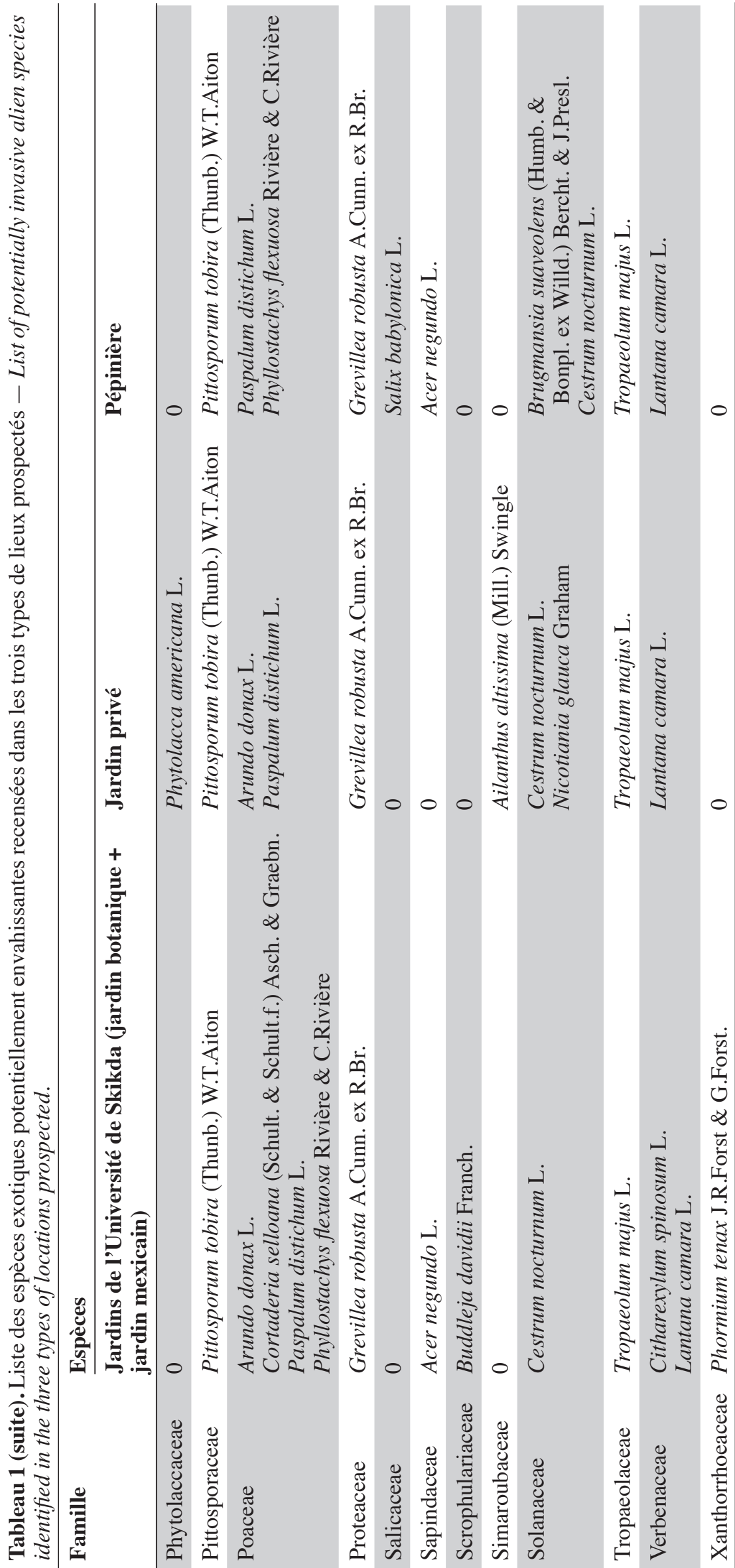
espèces et constituent le plus souvent des niches de propagation pour ces plantes, d'où elles peuvent effectivement s'échapper et coloniser les milieux naturels (Reichard \& White, 2001 ; Hulme, 2011). Les jardins privés et les pépinières sont aussi impliqués dans ces introductions et ces invasions (Reichard \& White, 2001; Smith et al., 2006). Le cas d'Oxalis pescaprae constitue un bon exemple de ces évasions à effets néfastes. Cette espèce, introduite en Algérie comme plante d'ornement, a été signalée par Munby (1847) aux environs d'Alger comme échappée d'un jardin et 41 ans plus tard, Battandier \& Trabut (1888) la signalent comme étant très commune et très envahissante à Alger. Enfin, après un siècle d'expansion, Quézel \& Santa (1963) la donnent comme très commune dans tout le Tell algérien.

\subsection{Caractéristiques biologiques des espèces potentiellement envahissantes de la wilaya de Skikda}

Les espèces potentiellement envahissantes sont essentiellement des plantes ligneuses, le maintien des individus sur une longue période permettrait l'adaptation aux différentes conditions environnementales et faciliterait la naturalisation (Fourdrigniez \& Meyer, 2008).

La multiplication végétative permet à la plante de devenir envahissante si elle est associée à une croissance rapide, comme c'est le cas pour l'Arundo donax.

Les espèces exotiques qui combinent deux modes de reproduction, sexuée et asexuée, peuvent se répandre rapidement dans les milieux pour devenir envahissantes (Albert et al., 2015). C'est notamment le cas d'Acacia dealbata, Acacia horrida, Acacia saligna et Ailanthus altissima qui se multiplient à la fois par graines et drageons. Si la multiplication sexuée assure l'établissement de 


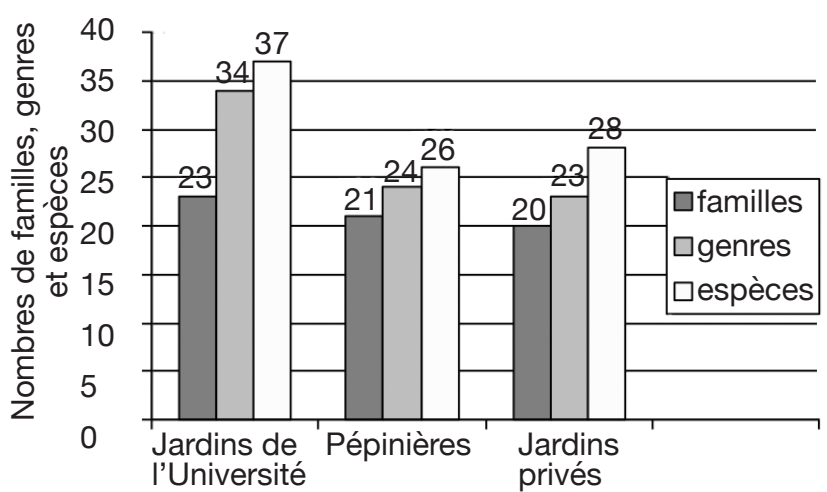

Lieux prospectés

Figure 2. Nombre de familles, genres et espèces recensés dans les pépinières, jardins privés et jardins de l'Université de Skikda (jardin botanique et jardin mexicain) - Number of families, genera and species identified in nurseries, private gardens and gardens of Skikda University (botanical garden and Mexican garden).

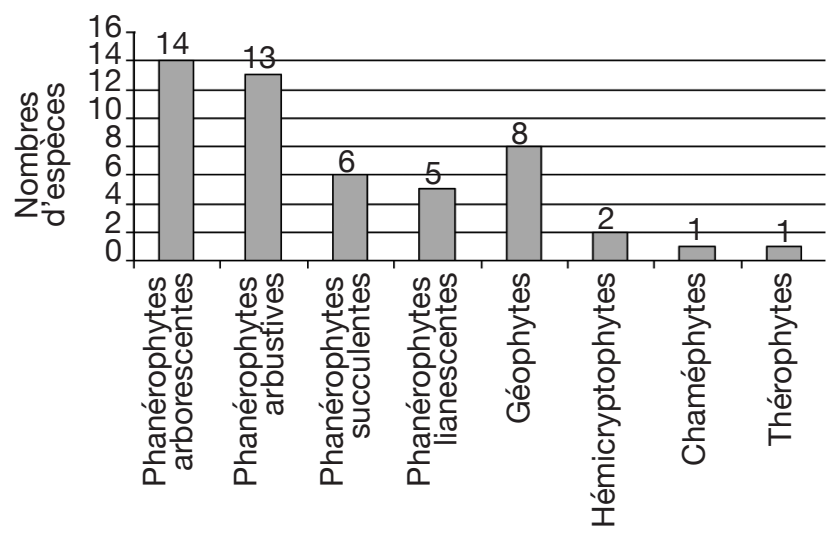

Types biologiques

Figure 3. Types biologiques identifiés - Biological types identified.

l'espèce dans des zones éloignées de la population mère, la multiplication végétative, quant à elle, assure le maintien et l'élargissement des populations établies, contribuant énormément à la production de formations denses et serrées (Dong et al., 2006). La conception de stratégies de contrôle efficaces repose en grande partie sur la connaissance de la contribution relative de la multiplication végétative et de la reproduction sexuée à la dispersion et l'établissement des plantes exotiques (Albert et al., 2015).

L'absence de fructification et, par conséquent, de graines chez certaines espèces cultivées dans les jardins et les pépinières, peut être expliquée par l'utilisation de plantes dioïques nécessitant la présence d'individus mâle et femelle pour la réalisation de la reproduction sexuée. C'est le cas de Citharexylum spinosum, Cortaderia selloana et Salix babylonica représentées chez nous par des individus femelles et de l'Acer negundo représentée par des individus mâles.

La dormance des graines, nécessitant des conditions particulières pour être levée, peut également être à l'origine de l'absence de germination et donc de plantules ; ce phénomène est observé chez de nombreuses espèces végétales appartenant aux différentes familles, comme par exemple la famille des Cactaceae où la dormance est rencontrée chez plusieurs genres, notamment l'Opuntia (Olivera-Carrillo et al., 2003 ; Altare et al., 2006). Les facteurs environnementaux et la mortalité des plantules à un stade très précoce après la germination peuvent aussi expliquer l'absence des plantules chez certaines espèces.

Différentes études ont mis en évidence la diversité des caractères biologiques rencontrée chez les espèces envahissantes et la difficulté d'établir un profil-type d'une espèce invasive (Roy, 1990 ; Lodge, 1993).

\subsection{Situation actuelle et prédictions d'invasions}

La synthèse bibliographique réalisée par Meddour \& El Mokni (2016) montre qu'une large portion $(45,29 \%)$ de plantes introduites en Algérie est naturalisée, avec environ $18 \%$ d'entre elles considérées comme envahissantes ou potentiellement envahissantes. À l'exception d'Acacia horrida, toutes les espèces naturalisées dans la zone de notre étude sont signalées envahissantes avérées dans plusieurs régions méditerranéennes (Brunel \& Tison, 2005 ; Capdevilla Argüelles et al., 2006 ; CelestiGrapow et al., 2009a ; Celesti-Grapow et al., 2009b ; Arianoutsou et al., 2010). Il conviendrait d'étudier de façon plus approfondie cette catégorie d'espèces afin de déterminer sa distribution spatiale au niveau de la wilaya, les milieux colonisés et les risques environnementaux liés à sa présence. Ces études pourront établir d'autres listes de références, comme la liste noire d'espèces envahissantes ayant un impact environnemental élevé, notamment sur la biodiversité, ou encore la liste grise d'espèces envahissantes ayant un impact environnemental modéré (Vanderhoeven et al., 2006).

De jeunes populations de Tradescantia fluminensis stabilisées de manière durable ont été retrouvées dans certains points de la commune de Skikda, notamment à la cité Larbi Ben M'Hidi. Cette espèce en voie de naturalisation dans notre région figure sur la liste des espèces à éradiquer en urgence en Espagne (Capdevilla Argüelles et al., 2006).

Quelques espèces non naturalisées ayant une multiplication végétative naturelle (production de drageons, stolons, rejets, tubercules, bulbes, etc.) sont également considérées comme envahissantes avérées en Méditerranée. Tel est le cas d'Acer negundo, Agave 
Tableau 2. Reproduction végétative, fructification, apparition de plantules issues des graines et degré de naturalisation des espèces potentiellement envahissantes - Vegetative reproduction, fruiting, seedling emergence from seeds and degree of naturalization of potentially invasive species.

\begin{tabular}{|c|c|c|c|c|}
\hline Espèce & $\begin{array}{l}\text { Reproduction végétative } \\
\text { naturelle }\end{array}$ & Fructification & $\begin{array}{l}\text { Nouvelles plantules } \\
\text { issues des graines }\end{array}$ & Naturalisation \\
\hline Acacia dealbata & Drageons & + & + & Non \\
\hline Acacia farnesiana & Drageons & + & - & Non \\
\hline Acacia horrida & Drageons & + & + & Oui \\
\hline Acacia saligna & Drageons & + & + & Oui \\
\hline Acer negundo & Rejets & - & - & Non \\
\hline Agave americana & Rhizomes et drageons & + & Non évalué & Non \\
\hline Ailanthus altissima & Drageons & + & + & Oui \\
\hline Arundo donax & Rhizomes & - & - & Oui \\
\hline Austrocylindropuntia subulata & Fragments de tiges & + & - & Oui \\
\hline Bauhinia variegata & - & + & - & Non \\
\hline Brugmansia suaveolens & Fragments de tiges & - & - & Non \\
\hline Buddleja davidii & - & - & - & Non \\
\hline Carpobrotus edulis & Stolons & + & - & Oui \\
\hline Cestrum nocturnum & Drageons & + & - & Non \\
\hline Chasmanthe floribunda & Bulbes & + & + & Non \\
\hline Cinnamomum camphora & - & + & + & Non \\
\hline Citharexylum spinosum & - & - & - & Non \\
\hline Cortaderia selloana & Fragments de rhizomes (rares) & - & - & Non \\
\hline Cryptostegia grandiflora & - & + & - & Non \\
\hline Cylindropuntia imbricata & Fragments de tiges & + & - & Non \\
\hline Eugenia uniflora & - & + & + & Non \\
\hline Ficus microcarpa & - & + & - & Non \\
\hline Grevillea robusta & - & + & + & Non \\
\hline Ipomea indica & Rejets et fragments de tiges & - & - & Non \\
\hline Jacaranda mimosifolia & - & + & - & Non \\
\hline Lantana camara & $\begin{array}{l}\text { Drageons, rejets, marcottage } \\
\text { naturel }\end{array}$ & + & - & Non \\
\hline Leucaena leucocephala & - & + & - & Non \\
\hline Lonicera japonica & Rejets & + & - & Non \\
\hline Macfadyena unguis-cati & Racines tubérisées & + & + & Non \\
\hline Melia azedarach & Drageons & + & - & Non \\
\hline Nicotiana glauca & - & + & + & Oui \\
\hline $\begin{array}{l}\text { Opuntia ficus-indica var. } \\
\text { amyclaea }\end{array}$ & Fragments de tiges & + & - & Oui \\
\hline $\begin{array}{l}\text { Opuntia ficus-indica var. } \\
\text { ficus-indica }\end{array}$ & Fragments de tiges & + & - & Oui \\
\hline Opuntia monacantha & Fragments de tiges & + & - & Non \\
\hline Opuntia stricta & Fragments de tiges & + & - & Oui \\
\hline Paspalum distichum & Rhizomes et stolons & - & - & Oui \\
\hline
\end{tabular}


Tableau 2 (suite). Reproduction végétative, fructification, apparition de plantules issues des graines et degré de naturalisation des espèces potentiellement envahissantes - Vegetative reproduction, fruiting, seedling emergence from seeds and degree of naturalization of potentially invasive species.

\begin{tabular}{|c|c|c|c|c|}
\hline Espèce & $\begin{array}{l}\text { Reproduction végétative } \\
\text { naturelle }\end{array}$ & Fructification & $\begin{array}{l}\text { Nouvelles plantules } \\
\text { issues des graines }\end{array}$ & Naturalisation \\
\hline Phormium tenax & Rhizomes & + & - & Non \\
\hline Phyllostachys flexuosa & Rhizomes & - & - & Non \\
\hline Phytolacca americana & Rejets & + & + & Non \\
\hline Pittosporum tobira & - & + & - & Non \\
\hline Psidium cattleianum & - & + & - & Non \\
\hline Psidium guajava & - & + & - & Non \\
\hline Robinia pseudoacacia & Drageons & + & Non évalué & Non \\
\hline Salix babylonica & Fragments de tiges & - & - & Non \\
\hline Senecio angulatus & Drageons & + & - & Non \\
\hline Stillingia sebifera & - & + & + & Non \\
\hline Syzygium jambolanum & - & + & - & Non \\
\hline Tradescantia fluminensis & Stolons & + & - & En cours \\
\hline Tradescantia zebrina & Stolons & + & - & Non \\
\hline Tropaeolum majus & - & + & + & Oui \\
\hline Zantedeschia aethiopica & Rhizomes & + & - & En cours \\
\hline
\end{tabular}

+ : présence - presence ; -: absence - absence.

americana, Cortaderia selloana, Cylindropuntia imbricata, Ipomea indica, Lantana camara, Lonicera japonica,Meliaazedarach, Opuntiamonacantha,Robinia pseudo-acacia, Senecio angulatus et Tradescantia zebrina (Brunel \& Tison, 2005 ; Capdevilla Argüelles et al., 2006 ; Celesti-Grapow et al., 2009a ; Podda et al., 2011 ; Terrin et al., 2014). Dans la zone d'étude, leur présence est limitée pour l'instant aux périmètres de culture, mais elles ont de fortes chances d'échapper des lieux de plantations pour coloniser les milieux voisins possédant des caractéristiques édaphiques similaires. Le risque est d'autant plus important pour les espèces ayant une reproduction sexuée efficace permettant la régénération et la croissance des plantules jusqu'à un stade assez avancé, reflétant leur grande adaptation aux conditions environnementales. Cette reproduction permet l'installation de l'espèce dans des zones plus éloignées des pieds mères. Dans cette catégorie, nous trouvons quelques espèces considérées comme envahissantes dans certains pays méditerranéens, dont Acacia dealbata, Cinnamomum camphora et Phytolacca americana sont les plus remarquables (Capdevilla Argüelles et al., 2006). D'après Branquart (2012), il faut être particulièrement vigilant à la production de formations denses par les espèces clonales, à la dispersion des graines et à l'installation de plantules à distance des populations d'origine. Les futures éventuelles naturalisations et peut-être même invasions dans la région de Skikda émergeront probablement à partir de ces deux catégories.

Selon Kolar \& Lodge (2001), les probabilités d'invasion augmentent si la plante possède des antécédents d'invasion et une reproduction végétative. Reichard \& Hamilton (1997) recommandent d'interdire l'introduction aux États-Unis des espèces signalées comme hautement envahissantes dans d'autres régions du monde. La mise en place d'un plan de surveillance au sein des jardins botaniques et des arboretums permet d'identifier les espèces invasives émergentes susceptibles de se répandre dans les milieux naturels (Branquart, 2012).

\subsection{Limites de la recherche}

L'absence de publications concernant les espèces envahissantes et potentiellement envahissantes cultivées en Algérie, au Maroc et en Tunisie nous a empêchés de réaliser des comparaisons à l'échelle nationale ou maghrébine.

\section{CONCLUSIONS}

Cette étude a permis de mettre en évidence l'existence, dans le répertoire horticole de la wilaya de Skikda, d'une cinquantaine d'espèces végétales considérées comme 
envahissantes dans différentes régions du monde et potentiellement envahissantes sur notre territoire. Pour la première fois dans la région, la liste préliminaire de ces espèces est établie. Elle met également le point sur leur degré de naturalisation, en identifiant 2 espèces en voie de naturalisation et 11 espèces naturalisées. Cette flore devrait faire l'objet de recherches spécifiques afin de déterminer sa répartition spatiale et son évolution, la nature des milieux colonisés, les impacts exercés sur la végétation indigène et de détecter les éventuelles invasions. Ces recherches permettront d'établir d'autres listes de références comme les listes noire et grise.

Le recensement et la connaissance des espèces potentiellement envahissantes devraient permettre la mise en place d'un plan d'action visant à contrôler leur culture et leur commercialisation. Les collections végétales exotiques sont souvent soumises à un changement permanent dû essentiellement aux nouvelles introductions et aux pertes, d'où la nécessité de réaliser des suivis périodiques dans les différentes exploitations horticoles permettant l'actualisation des listes et l'élaboration de stratégies de contrôle efficaces.

\section{Bibliographie}

Albert A. et al., 2015. Strategies for a successful plant invasion: the reproduction of Phragmites australis in north-eastern North America. J. Ecol., 103, 15291537.

Altare M. et al., 2006. Stimulation and promotion of germination in Opuntia ficus-indica seeds. J. Prof. Assoc. Cactus Dev., 8, 91-100.

APG III (The Angiosperm Phylogeny Group), 2009. An update of the angiosperm phylogeny group classification for the orders and families of flowering plants: APG III. Bot. J. Linn. Soc., 161, 105-121.

Arianoutsou M. et al., 2010. The alien flora of Greece: taxonomy, life traits and habitat preferences. Biol. Invasions, 12, 3525-3549.

Battandier J.A. \& Trabut L.C., 1888. La flore de l'Algérie. Dicotylédones. Paris : Libraire Savy.

Branquart É., 2012. Arbres et arbustes exotiques: une nouvelle vague d'envahisseurs? For. Wallonne, 120, 42-58.

Brunel S. \& Tison J.M., 2005. A method of selection and hierarchization of the invasive and potentially invasive plants in continental Mediterranean France. In: Brunel S., ed. Proceedings of the International workshop: invasive plants in Mediterranean type regions of the world, 25-27 May 2005, Mèze, France. Strasbourg, France: Council of Europe Publishing, 27-36.

Bruni G. et al., 2006. Botanica. Encyclopédie de botanique et d'horticulture. Paris : Éditions Place des Victoires.

Capdevilla Argüelles L., Iglesias Garcia A., Orueta J.F. \& Zilleti B., 2006. Especies exoticas invasoras: diagnostico y bases para la prevencion y el manejo. Madrid: Naturaleza y Parques Nationales, série Técnica.

Celesti-Grapow et al., 2009a. Plant invasion in Italy - an overview. Roma: Palombi \& Partners srl.

Celesti-Grapow et al., 2009b. Inventory of the non-native flora of Italy. Plant Biosyst., 143, 386-430.

Cullen J., Knees S.G. \& Cubey H.S., 2011. The European garden flora, Alismataceae to Orchidaceae. $2^{\text {nd }}$ ed. Cambridge, UK: Cambridge University Press.

De Belair G. \& Sameraoui B., 2000. L'éco-complexe des zones humides de Beni-Belaid: un projet de réserve naturelle. Sci. Technol., 14, 114-124.

Dehnen-Schmutz K., Touza A., Perrings C.\& Williamson M., 2007. The horticultural trade and ornamental plant invasions in Britain. Conserv. Biol., 21, 224-231.

Dong M. et al., 2006. Role of sexual reproduction in the spread of an invasive clonal plant Solidago canadensis revealed using intersimple sequence repeat markers. Plant Species Biol., 21(1), 13-18.

Emberger L., 1955. Une classification biogéographique des climats. Recl. Trav. Lab. Bot. Géol. Zool. Fac. Sci. Univ. Montpellier, 7, 3-43.

Farah A.K., 2014. Changement climatique ou variabilité climatique dans l'Est algérien. Thèse de magistère: Université Constantine 1 (Algérie).

Fourdrigniez M. \& Meyer J.-Y., 2008. Liste et caractéristiques des plantes introduites naturalisées et envahissantes en Polynésie française. Contribution à la Biodiversité de Polynésie française $n^{\circ} 17$. Papeete : Délégation à la Recherche.

Hequet V., Le Corre M., Rigault F. \& Blanfort V., 2009. Les espèces exotiques envahissantes de Nouvelle-Calédonie. Nouméa : IRD.

Hulme P.E., 2011. Addressing the threat to biodiversity from botanic gardens. Trends Ecol. Evol., 26(4), 168174.

Kolar C.S. \& Lodge D.M., 2001. Progress in invasion biology: predicting invaders. Trends Ecol. Evol., 16(4), 199-204.

Le Floc'h E., Le Houerou H.N. \& Mathez J., 1990. History and patterns of plant invasion in Northen Africa. In: Di Castri F., Hansen A.J. \& Debussche M., eds. Biological invasions in Europe and the Mediterranean Basin. Dordrecht, The Netherlands: Kluwer Academic Publishers, 105-133.

Lodge D.M., 1993. Biological invasions: lessons for ecology. Trends Ecol.Evol., 8, 133-137.

Magnanon S., Geslin J., Lacroix P. \& Zambettakis C., 2008. Examen du statut d'indigénat et du caractère invasif des plantes vasculaires de Basse-Normandie, Bretagne et Pays de la Loire. Propositions d'une première liste de plantes invasives et potentiellement invasives pour ces trois régions. Bull. Conservatoire Bot. Natl Brest E.R.I.C.A., 21, 73-104.

Meddour R. \& El Mokni R., 2016. État de l'art sur les plantes envahissantes ou à caractère invasif introduites 
en Algérie et en Tunisie. In: Actes de la Conférence XV OPTIMA Meeting, 6-11 juin 2016, Montpellier, France.

Meghzili H., 2015. Modèles d'aménagement et d'urbanisation des zones d'expansion touristique de la wilaya de Skikda (Algérie). Thèse de doctorat: Université de Bretagne Occidentale, Brest (France).

Moran V.C., Hoffmann J.H. \& Zimmermann H.G., 2013. 100 years of biological control of invasive alien plants in South Africa: history, practice and achievements. S. Afr. J. Sci., 109(9/10), 1-6.

Muller S., 2000. Les espèces végétales invasives en France : bilan des connaissances et propositions d'actions. Rev. Écol. (Terre et Vie), 7, 53-69.

Munby G., 1847. Flore de l'Algérie ou catalogue des plantes indigènes. Paris : J.-B. Baillière.

Odenwald N.G.\& Turner J.R.,2006.Identification, selection and use of southern plants for landscape design. Baton Rouge, LA, USA: Claitor's Publishing.

Olivera-Carrillo Y. et al., 2003. Germination of the hard seed coated Opuntia tormentosa S.D., a cacti from México Valley. J. Arid Environ., 55, 29-42.

OTA (Office of Technology and Assessment, U.S. Congress), 1993. Harmful non-indigenous species in the United States, OTA-F-565. Washington, DC: U.S. Government Printing Office.

Podda L. et al., 2011. Comparison of the invasive alien flora in continental islands: Sardinia (Italy) and Balearic islands (Spain). BMC Biol., 22, 31-45.

Quézel P. \& Santa S., 1963. Nouvelle flore d'Algérie et des régions désertiques méridionales. Tome 2. Paris : Centre National de la Recherche Scientifique.

Raunkiaer C., 1934. The life forms of plants and statistical plant geography. Oxford, UK: Clarendon Press.

Reichard S.H. \& Hamilton C.W., 1997. Predicting invasion of woody plants introduced into North America. Conserv. Biol., 11(1), 193-203.

Reichard S.H. \& White P., 2001. Horticulture as a pathway of invasive plant introductions in the United States. Bioscience, 51(2), 103-113.

Roy J., 1990. In search of the characteristics of plant invaders. In: Di Castri F., Hansen A.J. \& Debussche M., eds. Biological invasions in Europe and the Mediterranean basin. Dordrecht, The Netherlands: Kluwer Academic Publishers, 335-352.

Shine C., William N. \& Gunbdling L., 2000. A guide to designing legal and institutional frameworks on alieninvasive species. Droit et politique de l'environnement $\mathrm{n}^{\circ} 40$. Gland, Switzerland: UICN.

Smith R.M. et al., 2006. Urban domestic gardens (IX): composition and richness of the vascular plant flora and implication for native biodiversity. Biol. Conserv., 29(3), 312-322.

Terrin E., Diadema K. \& Fort N., 2014. Stratégies régionales relatives aux espèces végétales exotiques envahissantes en Provence-Alpes-Côte d'Azur et son plan d'action. Chambéry, France: Conservatoire Botanique National Alpin; Porquerolles, France: Conservatoire Botanique National Méditerranéen de Porquerolles.

Vanderhoeven S., Branquart É., Grégoire J.-C. \& Mahy G., 2006. Les espèces exotiques envahissantes - dossier scientifique réalisédans le cadre de l'élaboration du rapport analytique 2006-2007 sur l'état de l'environnement wallon, http://environnement.wallonie.be/eew, (07/09/2018).

Véla E., 2013. Notes sur les cactus du genre Opuntia s. 1. en Algérie et en Tunisie. In : Dobignard A. \& Chatelain C., éds. Addenda-Notes. Index synonymique de la flore d'Afrique du Nord. Genève: Conservatoire et Jardin Botaniques, 376-379.

Véla E., Rebbas K., Meddour R. \& de Belair G., 2013. Note sur quelques xénophytes nouveaux pour l'Algérie (et la Tunisie). In: Dobignard A. \& Chatelain C., eds. Addenda - Notes. Index synonymique de la flore d'Afrique du Nord. Genève: Conservatoire et Jardin Botaniques, 372-376.

Vilà M. et al., 2015. Explaining the variation in impacts of non-native plants on local-scale species richness: the role of phylogenetic relatedness. Glob. Ecol. Biogeogr., 24, 139-146.

Weber E., 2017. Invasive plant species of the world: a reference guide to environmental weeds. $2^{\text {nd }}$ ed. Wallingford, UK; Boston, MA, USA: CABI Publishing.

(46 réf.) 